BULLETIN (New Series) OF THE

AMERICAN MATHEMATICAL SOCIETY

Volume 33, Number 2, April 1996

\title{
ON SOME APPLICATIONS OF AUTOMORPHIC FORMS TO NUMBER THEORY
}

\author{
DANIEL BUMP, SOLOMON FRIEDBERG, AND JEFFREY HOFFSTEIN
}

\begin{abstract}
A basic idea of Dirichlet is to study a collection of interesting quantities $\left\{a_{n}\right\}_{n \geq 1}$ by means of its Dirichlet series in a complex variable $w$ : $\sum_{n \geq 1} a_{n} n^{-w}$. In this paper we examine this construction when the quantities $a_{n}$ are themselves infinite series in a second complex variable $s$, arising from number theory or representation theory. We survey a body of recent work on such series and present a new conjecture concerning them.
\end{abstract}

\section{INTRODUCTION}

The object of this paper is to give a survey of a body of recent work applying methods from automorphic forms to problems in number theory. Generalizing this work, we shall also formulate a new conjecture concerning Langlands $L$-functions, which implies such results as the Lindelöf Hypothesis in twisted aspect. This new conjecture arises from the work of various subsets of the three authors and D. Goldfeld, and the authors gratefully acknowledge the influence of Prof. Goldfeld's ideas on this conjecture and on their work.

The analytic underpinning of these applications is a basic idea which goes back to Dirichlet: suppose one has a collection of interesting quantities $\left\{a_{n}\right\}_{n \geq 1}$ (interesting, that is, from the viewpoint of arithmetic or representation theory). Take these quantities and build a Dirichlet series out of them:

$$
D(w)=\sum_{n=1}^{\infty} \frac{a_{n}}{n^{w}}
$$

If the analytic properties of $D(w)$ in a complex variable $w$ can be discovered, they may often be used to derive information about the $a_{n}$.

For example, take

$$
a_{n}=\Lambda(n)= \begin{cases}\log p & \text { if } n=p^{k} \text { for some prime } p \text { and integer } k \geq 1 \\ 0 & \text { otherwise }\end{cases}
$$

Then

$$
D(w)=\sum \frac{\Lambda(n)}{n^{w}}=-\frac{\zeta^{\prime}(w)}{\zeta(w)}
$$

1991 Mathematics Subject Classification. Primary 11F66, Secondary 11F70, 11M41, 11N75.

Research supported by NSA grant MDA904-95-H-1053 (Friedberg) and by NSF grants DMS9346517 (Bump) and DMS-9322150 (Hoffstein).

(C)1996 American Mathematical Society 
where $\zeta(w)=\sum_{n=1}^{\infty} n^{-w}$ is the Riemann zeta function. In this case, $D(w)$ converges for $\operatorname{Re}(w)>1$ and has a simple pole at $w=1$ (inherited from the simple pole of $\zeta(w))$. Since the sum over those $n$ which are products of powers of a fixed set of primes $p_{1}, \cdots, p_{r}$ is given by

$$
\sum_{i=1}^{r} \sum_{k \geq 1} \frac{\log p_{i}}{p_{i}^{k w}}=\sum_{i=1}^{r}\left(\log p_{i}\right) p_{i}^{-w}\left(1-p_{i}^{-w}\right)^{-1}
$$

convergent for $\operatorname{Re}(w)>0$, the existence of a pole at $w=1$ implies that there are infinitely many primes.

Dirichlet invented Dirichlet $L$-series in order to obtain the analytic continuation of a somewhat more refined series. Choose any modulus $q$ and an integer $a$ such that $(a, q)=1$. Let

$$
D(w, a, q)=\sum_{n \equiv a \bmod q} \frac{\Lambda(n)}{n^{w}} .
$$

Then Dirichlet showed that $D(w, a, q)$ also has a pole at $w=1$, implying the existence of infinitely many primes $p \equiv a \bmod q$.

These are examples of some of the simplest applications of the analytic study of Dirichlet series: if $D(w)$ has a pole in $w$, then infinitely many of the $a_{n}$ must be non-zero. By taking the analytic argument further, more can often be learned. For example, in these cases by analytically continuing past $\operatorname{Re}(w)=1$ and obtaining a thin region free of zeroes near $\operatorname{Re}(w)=1$, one may establish mean value estimates. Stating these in asymptotic form (as $x \rightarrow \infty$ ), one has first the Prime Number Theorem

$$
\sum_{n<x} \Lambda(n) \sim x
$$

and second Dirichlet's theorem on primes in an arithmetic progression

$$
\sum_{\substack{n<x \\ n \equiv a \bmod q}} \Lambda(n) \sim \frac{1}{\phi(q)} x .
$$

In this paper we will not be concerned with how such mean value results are established. But it is worthwhile to note that they are always in principle obtainable, given enough analytic information.

We are left with the questions of describing interesting choices of the coefficients $a_{n}$ and of indicating how such analytic information in $w$ may be obtained. One large family of interesting Dirichlet series was described by Langlands [La]. These series, each associated to an automorphic form and some additional data, are series with Euler product and, conjecturally, with analytic continuation and functional equation as well. For an excellent survey, see Gelbart [Ge]. The study of the Langlands Conjectures is ongoing and related to many fundamental problems in number theory, such as Artin's Conjecture. In this paper we shall suggest a new family of interesting Dirichlet series. We shall make the case that for many different types of $\left\{a_{n}\right\}$, which are themselves infinite series in a second complex variable $s(!)$, the construction of Dirichlet gives a series

$$
D(s, w)=\sum_{n} \frac{a_{n}(s)}{n^{w}}
$$


in two complex variables $s, w$ with nice properties, which moreover may be used to obtain number-theoretic or representation-theoretic information. Though these series are typically not Euler products, their behavior in $w$ may be studied by using variants on the Rankin-Selberg method from the theory of automorphic forms. As for precisely which coefficients $a_{n}(s)$ and as for what the Rankin-Selberg method is and how it is used, it is useful to begin with some examples.

\section{First Cases: Sums of Dirichlet $L$-SERIES}

Let us begin with the case that the $a_{n}(s)$ are series of classical number-theoretic interest, namely, quadratic Dirichlet $L$-series. For $n$ a fundamental discriminant, let $L\left(s, \chi_{n}\right)$ be the $L$-series associated to the quadratic field $\mathbb{Q}(\sqrt{n})$. That is, $\chi_{n}$ is defined by the quadratic residue symbol $\chi_{n}(m)=\left(\frac{n}{m}\right)$, and $L\left(s, \chi_{n}\right)$ is given by the (analytic continuation of the) Dirichlet series

$$
L\left(s, \chi_{n}\right)=\sum \frac{\chi_{n}(m)}{m^{s}}
$$

thus the Dedekind zeta function associated to $\mathbb{Q}(\sqrt{n})$ is precisely $\zeta(s) L\left(s, \chi_{n}\right)$. Then the quantities $L\left(s, \chi_{n}\right)$ for fixed $s$ but varying $n$ carry interesting information. For example, at $s=1$ the value $L\left(1, \chi_{n}\right)$ encodes $h(n)$, the class number of the ring of integers of $\mathbb{Q}(\sqrt{n})$, and the regulator if $n>0$. Gauss made some conjectures about the mean value of $L\left(1, \chi_{n}\right)$ as $n$ varies over either positive or negative discriminants that were eventually proved by Siegel and others (see $[\mathrm{G}-\mathrm{H}]$ for a brief history). Alternatively, one could consider $s=1 / 2$. The best-known upper bound for $L\left(1 / 2, \chi_{n}\right)$ is due to Burgess [Bu]:

$$
L\left(1 / 2, \chi_{n}\right) \ll|n|^{\frac{3}{16}+\epsilon},
$$

but the $n$-analog of the Lindelöf Hypothesis implies that the true upper bound should be

$$
L\left(1 / 2, \chi_{n}\right) \ll|n|^{\epsilon} .
$$

The value $L\left(1 / 2, \chi_{n}\right)$ has no known arithmetic significance, but the question of the true size of $L\left(1 / 2, \chi_{n}\right)$ seems to be a very deep one, and there are also a number of applications where it is desirable to have the best possible upper bound. Also, it is of interest to determine the nature of the mean value of $L\left(1 / 2, \chi_{n}\right)$, for comparison with the mean value predicted by the Lindelöf Hypothesis.

As suggested in the introduction, it is then natural to introduce the (two) functions of two complex variables $s, w$ :

$$
D_{ \pm}(s, w)=\sum_{ \pm n>0} \frac{L\left(s, \chi_{n}\right)}{|n|^{w}}
$$

where in the sum here $n$ varies over fundamental discriminants whose sign is specified by the index \pm . This series was considered by Goldfeld and Hoffstein in [G-H]. They studied its analytic properties by linking it to certain automorphic forms: Eisenstein series of half-integral weight. Let $z=x+i y$ be in the Poincaré upper half plane, and $j(\gamma, z)$ be the classical theta multiplier

$$
j(\gamma, z)=\epsilon_{d}^{-1}\left(\frac{c}{d}\right)(c z+d)^{1 / 2}
$$


where $\gamma=\left(\begin{array}{ll}a & b \\ c & d\end{array}\right)$ is in the congruence subgroup $\Gamma_{0}(4)$ of $S L_{2}(\mathbb{Z}), \epsilon_{d}=1$ if $d \equiv 1$ $(\bmod 4), \epsilon_{d}=i$ if $d \equiv 3(\bmod 4),\left(\frac{c}{d}\right)$ is a modified Kronecker symbol as in Shimura [Shi], and $(c z+d)^{1 / 2}$ is chosen so that $\left|\arg \left((c z+d)^{1 / 2}\right)\right|<\frac{\pi}{2}$. The half-integral weight Eisenstein series is defined by

$$
E_{\infty}(z, s)=\zeta(4 s) \sum_{\gamma \in \Gamma_{\infty} \backslash \Gamma_{0}(4)} j(\gamma, z)^{-1} \operatorname{Im}(\gamma z)^{s} .
$$

This converges for $\operatorname{Re}(s)>3 / 4$ and is an automorphic form of weight $1 / 2$ on $\Gamma_{0}(4)$.

Both $E_{\infty}$ and the translate of $E_{\infty}$ to the cusp 0,

$$
E_{0}(z, s)=z^{-1 / 2} E_{\infty}(-1 / 4 z, s)
$$

are invariant under shifts in $z$, and so have Fourier expansions. The key point (essentially due to Maass [Ma]; for an exposition see [Ho, Section 1]) exploited in $[\mathrm{G}-\mathrm{H}]$ is that the $n$-th Fourier coefficient of $E_{0}$ involves the quadratic $L$-series $L\left(2 s, \chi_{n}\right)$. To be more precise, if

$$
E_{0}(z, s)=\sum a_{n}(s, y) e(n x)
$$

with $e(n x)=\exp (2 \pi i n x)$, then for $n \neq 0$

$$
a_{n}(s, y)=A_{n}(s) W_{\operatorname{sgn}(n)}(|n| y, s),
$$

where $W_{\operatorname{sgn}(n)}(y, s)$ is a complicated Whittaker function depending on the sign of $n$ and $A_{n}(s)$ is a Dirichlet series. If $n=n_{0} n_{1}^{2}$ with $n_{0}$ squarefree, then

$$
A_{n}(s)=L_{2}\left(2 s, \chi_{n_{0}}\right) b_{n}(s)
$$

where $L_{2}\left(2 s, \chi_{n_{0}}\right)$ is the quadratic $L$-series associated to the field $\mathbb{Q}\left(\sqrt{n_{0}}\right)$ with the Euler factor corresponding to 2 removed, and $b_{n}(s)$ is a Dirichlet polynomial which equals 1 if $n_{1}=1$ and whose complexity grows as $n_{1}$ increases.

Following Hecke, one may take the classical Mellin transform of $E_{0}(z, s)$ in a new complex variable $w$ (with some technical steps to insure convergence). This yields the series

$$
I_{+}(s, w) \sum_{n>0} A_{n}(s) n^{-w}+I_{-}(s, w) \sum_{n<0} A_{n}(s)|n|^{-w}
$$

where

$$
I_{ \pm}(s, w)=\int_{0}^{\infty} W_{ \pm}(y, s) y^{w-1} d y .
$$

The integrals defining $I_{ \pm}$are convergent for $\operatorname{Re}(s), \operatorname{Re}(w)$ sufficiently large and have meromorphic continuation to all of $\mathbb{C}^{2}$; they are not products of $\Gamma$-functions, but rather products of $\Gamma$-functions and hypergeometric functions; see $[\mathrm{G}-\mathrm{H}]$. The Hecke integral implies that, remarkably, the expression (1.3) satisfies a functional equation in $w$. Going through some sieving to eliminate all but squarefree terms and some asymptotic analysis of the Whittaker functions to separate positive and negative discriminants, one may obtain the analytic properties of $D_{ \pm}$. 
As an application, one gets, as in $[\mathrm{G}-\mathrm{H}]$, that for any $s$ with $\operatorname{Re}(s) \geq 1 / 2$,

$$
\sum_{\substack{0< \pm n<x \\ n \text { squarefree }}} L_{2}\left(s, \chi_{n}\right)=c_{1}(s) x+c_{2}(s, \pm) x^{3 / 2-s}+c_{3}(s) x \log x+\mathrm{O}\left(x^{\theta(s)+\epsilon}\right) .
$$

Here $c_{2}(s, \pm)=0$ for $\operatorname{Re}(s) \geq 1, c_{3}(s)=0$ if $s \neq 1 / 2$, and if $s=1 / 2$, then $\theta(s)=19 / 32$. In the past, as in Siegel [Si], this was treated by writing $L_{2}\left(s, \chi_{n}\right)$ as a finite sum using the approximate functional equation, interchanging the order of summation, and using estimates for exponential sums; the best result obtained by this method, due to Jutila [Ju], is $\theta(1 / 2)=3 / 4$.

Similar techniques allow one to study the series

$$
D_{ \pm}(s, w ; \chi)=\sum_{ \pm n>0} \frac{L\left(s, \chi \chi_{n}\right)}{|n|^{w}}
$$

where $\chi$ is any fixed Dirichlet character. Rather than summing over fundamental discriminants, one may sum over all $n$ of the given sign but define $L\left(s, \chi \chi_{n}\right)$ to match (1.2) when $n$ is a square times a fundamental discriminant. One may also pass to an analogous series for an arbitrary number field, where $\chi$ is now a Grössencharacter. Moreover, these series may also be studied by more exotic methods: they occur as the Whittaker-Fourier coefficients of certain Eisenstein series on the metaplectic double cover of $G L(3)$, and one may deduce their properties from this fact.

One may obtain a second example of a Dirichlet series in $w$ whose coefficients are themselves Dirichlet series in $s$ by considering the Rankin-Selberg convolution of $E_{0}(z, s)$ with itself. Traditionally, if $f, g$ are $G L(2)$ cusp forms with Fourier coefficients $a_{n}, b_{n}$, then the Rankin-Selberg convolution of $f$ with $g$ is an integral which represents the Dirichlet series

$$
L(w, f \times g)=\sum \frac{a_{n} \overline{b_{n}}}{n^{w}},
$$

and from which the analytic continuation of (1.5) may be deduced. (See [Bump] for a detailed survey of the Rankin-Selberg method.) Though the details have not yet been carried out, in this case such a convolution should give the analytic continuation of the two series

$$
\sum_{ \pm n>0} \frac{\left|L\left(s, \chi_{n}\right)\right|^{2}}{|n|^{w}}
$$

Such a continuation would imply formulas for the mean value of $\left|L\left(s, \chi_{n}\right)\right|^{2}$. For example, at $s=1 / 2$ one should find an estimate for

$$
\sum_{\substack{0< \pm n<x \\ n \text { squarefree }}}\left|L\left(1 / 2, \chi_{n}\right)\right|^{2}
$$

where the main term $C x(\log x)^{3}$ ( $C$ a non-zero constant) is larger than the error term by a power of $x$. A result of this form has been found by Jutila [Ju] by 
other methods, with a saving of a power of $\log x$ in the error term. Note that both examples above are consistent with the Lindelöf Hypothesis.

In both these examples, the application of the integral transforms (Hecke or Rankin-Selberg integrals) is more difficult than the standard situation, for the function $E_{0}(z, s)$ is not of rapid decay as $y \rightarrow \infty$ and this causes convergence problems. However, this may be overcome, as in Zagier [Za] or Lieman [Li1]; for higher rank groups the theory of truncations of such functions, though by no means simple, is well understood, due to the work of Arthur [Ar, Lemma 1.4].

\section{Further exAmples: $G L(2)$}

The series $L\left(s, \chi_{n}\right)$ can be thought of as the quadratic twists of the $G L(1) L$-series $\zeta(s)$. Can something similar be done in the case of a $G L(2) L$-series? Specifically, let $f$ be a newform with $L$-series $L(s, f)=\sum c_{m} m^{-s}$ and let

$$
L\left(s, f, \chi_{n}\right)=\sum c_{m} \chi_{n}(m) m^{-s}
$$

denote the twist of $L(s, f)$ by the quadratic character $\chi_{n}$. Suppose that these series are normalized to have functional equations under $s \mapsto 1-s$. Then, for a variety of applications, it is desirable to know that there are infinitely many distinct fundamental discriminants $n$ such that $L\left(1 / 2, f, \chi_{n}\right) \neq 0$; moreover, it is desirable to know that this remains true even if one imposes a finite number of local conditions on $n$. Similarly, there are applications where it is useful to know that $L\left(s, f, \chi_{n}\right)$ has a simple zero at $s=1 / 2$ for infinitely many such $n$.

This leads us to our next examples of Dirichlet series of the form (0.1), the series

$$
\sum_{\substack{ \pm n>0 \\ n \equiv a \bmod N}} \frac{L\left(s, f, \chi_{n}\right)}{n^{w}}
$$

and

$$
\sum_{\substack{ \pm m>0 \\ n \equiv a \bmod N}} \frac{L^{\prime}\left(s, f, \chi_{n}\right)}{n^{w}}
$$

for given $N$, and $(a, N)=1$. Here the sums are over fundamental discriminants. For example, if (2.1) can be shown to have a pole at $w=1$ when $s=1 / 2$, then $L\left(1 / 2, f, \chi_{n}\right) \neq 0$ for infinitely many $n \equiv a \bmod N$; while if $(2.2)$ has a pole at $w=1$ and $a$ is chosen so that $L\left(1 / 2, f, \chi_{n}\right)=0$ for all such $n$ (for example, because of the sign of the functional equation), then $L\left(s, f, \chi_{n}\right)$ has a simple zero at $s=1 / 2$ for infinitely many such $n$. Note that (2.2) is obtained by differentiating (2.1) with respect to $s$.

These series were first studied in a series of papers by the authors [BFH1][BFH3]. The basic idea of these papers is to form from $f$ an Eisenstein series $E_{f}(Z, s)$ of half integral weight on the Siegel upper half-space of genus two. This Eisenstein series may be viewed as a function on the metaplectic double cover of the symplectic similitude group $G S p(4)$; in this context it was first introduced by Gelbart and Piatetski-Shapiro in $[\mathrm{G}-\mathrm{P}]$. The existence of $E_{f}(Z, s)$ hinges on the observation that the double cover splits over an embedded copy of $G L(2)$. The authors 
then proceed to study the Whittaker-Fourier coefficients of $E_{f}(Z, s)$; these are certain analogues of classical Fourier coefficients, obtained by integrating $E_{f}$ against a character of the unipotent radical of a Borel subgroup of GSp(4). The authors show that the Whittaker-Fourier coefficients of $E_{f}(Z, s)$ may be expressed in terms of the twisted $L$-series $L\left(s, f, \chi_{n}\right)$; here the parameter $n$ is related to the character of the unipotent radical. Then, using an integral transform due to Novodvorsky [No], they are able to obtain the series (2.1) and (2.2) as sums of Whittaker-Fourier coefficients of $E_{f}$ and $\frac{\partial}{\partial s} E_{f}$. The properties of these series are thus linked to the properties of the automorphic form $E_{f}$, and the desired behavior is obtained. In particular one obtains information about the nonvanishing of $L^{\prime}\left(1 / 2, f, \chi_{n}\right)$ which is, due to the work of Kolyvagin [Ko], of arithmetic significance (cf. [BFH1, BFH3]). We note that, when applied to a non-metaplectic automorphic form on $G S p(4)$ with non-zero Whittaker-Fourier coefficients, the Novodvorsky transform gives a degree-four Langlands $L$-function with Euler product; it is another example of a "Rankin-Selberg integral".

At least over $\mathbb{Q}$, the mean values for these series may also be studied by traditional analytic number theoretic methods, averaging à la Siegel. This method yields the result

$$
\sum_{n \equiv a \bmod N, n \text { squarefree }} L^{\prime}\left(1 / 2, f, \chi_{n}\right) F(x / n)=c_{1} x+\mathrm{O}\left(x^{1-\delta}\right) .
$$

Here $F(x / n)$ is a smoothing function with compact support (for example, on [1,2]). This was obtained by Iwaniec $[\mathrm{Iw}]$ when $f$ is modular over $\mathbb{Q}$ of weight 2 . This followed a similar result of Murty and Murty [M-M] (unsmoothed) with a somewhat weaker error term. The averaging of a $G L(2)$ form was considerably more difficult than that of a $G L(1)$ form, and it is not clear if this approach can be extended beyond $G L(2)$. It is also not clear what happens when the base field is changed.

The analysis of series analogous to (2.1) and (2.2) may be carried out when $f$ is replaced by an arbitrary automorphic representation of $G L(2)$ over an arbitrary number field. Moreover, it is rather surprising that these series (or more precisely ones with coefficients identical to them except at finitely many primes) may be obtained by two other Rankin-Selberg type integrals! First, the authors (unpublished) have found a construction similar to the above, but where the metaplectic group $G S p(4)$ is replaced by the classical group $O(5)$ and the Whittaker-Fourier coefficients are replaced by certain coefficients called Bessel coefficients. (This is perhaps not surprising, as automorphic forms on these two groups are linked by the theta correspondence.) Second, a completely new construction, studied in $[\mathrm{F}-\mathrm{H}]$, is to consider the Rankin-Selberg convolution (1.5) of the (non-metaplectic) automorphic form $f$ on $G L(2)$ with a half-integral weight (metaplectic) Eisenstein series on $G L(2)$; that is, one studies

$$
L\left(s, f \times E_{0}(\cdot, \bar{w} / 2)\right) .
$$

If the Fourier coefficients of $f$ are $c_{n}$ then, by (1.2) and (1.5), this convolution gives the analytic continuation of a Dirichlet series which is roughly of the form

$$
\sum \frac{c_{n} L\left(w, \chi_{n}\right)}{n^{s}}
$$


Substituting the infinite sum (1.1) which represents the quadratic $L$-series $L\left(w, \chi_{n}\right)$ and interchanging the order of summation gives, remarkably, the series (2.1), with some shifts in $s, w$ (after appropriate sieving). Using this, Friedberg and Hoffstein $[\mathrm{F}-\mathrm{H}]$ proved a non-vanishing theorem for the $L$-series of an arbitrary cuspidal automorphic form on $G L(2)$ over any number field under quadratic twists. In the case of an imaginary quadratic field, such a result has applications to the construction of $\ell$-adic representations from certain modular forms; see the work of Harris, Soudry and Taylor [HST] and Taylor [Ta].

One naturally asks if this approach may be generalized. It has been carried over to $G L(2)$ automorphic forms over function fields by S. Gupta $[\mathrm{Gu}]$, who used it to obtain non-vanishing and mean value theorems for such $L$-series under quadratic twists. However, the higher rank case is more difficult. To carry out this construction for $f$ on $G L(r)$ with $r \geq 3$ (and so obtain the analogue of (2.1)) would require a Rankin-Selberg convolution for a (non-metaplectic) cusp form on $G L(r)$ with an Eisenstein series on the double cover of $G L(2)$. There does not yet exist a way of doing this when $r \geq 3$. A different generalization may be obtained; this is one of the constructions described in Section 5 below.

\section{A HIGHER RANK EXAMPLE}

In what ways may one seek generalizations of the two previous examples? One direction comes immediately to mind. Namely, one might hope to replace the standard $G L(1)$ or $G L(2) L$-series by a more general Langlands $L$-function (see Langlands [La], Borel [Bo], Gelbart [Ge]) and sum its quadratic twists. Surprisingly, the examples of Sections 1 and 2 exhaust the list of cases where such a sum has been analyzed in full detail. However, the case of the standard $L$-function associated to a $G L(3)$ automorphic representation is within reach, as we shall explain in this section. Then, in Section 4, we shall state a general conjecture and present some heuristic arguments suggesting that the situation is dramatically more complicated if the degree of the Euler product in $s$ is 4 or more.

In the constructions of Sections 1 and 2, automorphic objects (Eisenstein series) were exhibited whose Whittaker-Fourier coefficients are twisted $L$-functions. These coefficients were then "strung together" into the Dirichlet series $(0.1)$ by means of an integral transform. This raises the natural question (which is related to the $G L(3)$ example to be presented momentarily): how generally may one represent the twists of a given Langlands $L$-function, up to a finite number of Euler factors, as the coefficients of an automorphic form (more precisely, an Eisenstein series)? Coefficient here has a representation-theoretic meaning: the value of a unique functional, such as a Whittaker functional, depending on a character, which one varies to obtain the different twists. This can be accomplished in two general instances.

Let $\pi=\otimes \pi_{v}$ be a factorizable automorphic representation of $G L(m)$ over $\mathbb{Q}$ with unitary central character. Then attached to $\pi$ is an $L$-function, $L(s, \pi)$, which is a product of local $L$-functions $L_{v}\left(s, \pi_{v}\right)$, almost all given by degree $m$ Euler factors (see Langlands [La], Borel [Bo]). That is, $L(s, \pi)=\prod_{v} L\left(s, \pi_{v}\right)$, where the product is over the places $v$ of $\mathbb{Q}$ and, for almost all $v$,

$$
L_{v}\left(s, \pi_{v}\right)=\prod_{i=1}^{m}\left(1-\alpha_{i, v} v^{-s}\right)^{-1}
$$


Similarly, one may define the twisted $L$-function $L\left(s, \pi, \chi_{n}\right)$ as the product of the twisted factors, given for almost all $v$ by the formula

$$
L_{v}\left(s, \pi_{v}, \chi_{n}\right)=\prod_{i=1}^{m}\left(1-\alpha_{i, v} \chi_{n}(v) v^{-s}\right)^{-1}
$$

Then it has been shown by the authors [BFH4] that, in analogy to the situations when $m=1$ and $m=2$, the twists $L\left(s, \pi, \chi_{n}\right)$ do occur as Whittaker-Fourier coefficients for any $m \geq 3$ as well, this time of an Eisenstein series on the metaplectic double cover of $G S p(2 m)$ induced from $\pi$ on $G L(m)$. Similarly, it has been shown by Bump, Friedberg, and Furusawa $[\mathrm{BFF}]$ that these twists occur as Bessel coefficients of an Eisenstein series on the orthogonal group $S O(2 m+1)$ induced from $\pi$. (A similar relation between the Whittaker and Bessel coefficients of cuspidal automorphic representations in theta correspondence on these two groups was proved by Furusawa $[\mathrm{Fu}]$ using different techniques.)

One would like to assemble these coefficients into the series (0.1). As described in Section 2, working on the metaplectic double cover of $G S p(4)$, the authors accomplished this assembly when $m=2$ by applying an integral transform due to Novodvorsky [No], which in the non-metaplectic case gives a Rankin-Selberg integral representing the degree 4 "Spin" $L$-function associated to a generic automorphic form on $G S p(4)$. One is thus tempted to use higher degree "Spin" integrals. For $m=3,4,5$, Bump and Ginzburg [B-G] have given an integral which represents the "Spin" $L$-function of a non-metaplectic cusp form on $G S p(2 m)$. For $m=3$ the same integral works on the double cover (of $G S p(6)$ ), and it should thus lead to the analytic continuation of the series

$$
\sum_{ \pm n>0} \frac{L\left(s, \pi, \chi_{n}\right)}{|n|^{w}}
$$

for $\pi$ an automorphic form on $G L(3)$. This, in principle, should yield results of the form

$$
\sum_{0< \pm n, n \text { squarefree }} L\left(1 / 2, \pi, \chi_{n}\right) F(x / n)=c x+\mathrm{O}\left(x^{1 / 2+\epsilon}\right)
$$

for $\pi$ with unitary central character (and, in particular, non-vanishing theorems for quadratic twists, even at the center of the critical strip; compare BarthelRamakrishnan $[\mathrm{B}-\mathrm{R}])$. We believe this to be inaccessible by current averaging techniques. Also, considering non-cuspidal $\pi$ induced from a parabolic subgroup whose Levi factor is $G L(2) \times G L(1)$, one should be able to control sums such as

$$
\sum_{ \pm n<x} L\left(1, \chi_{n}\right) L\left(1 / 2, E^{(n)}\right)
$$

where $L\left(1, \chi_{n}\right)$ is expressible in terms of the arithmetic of $\mathbb{Q}(\sqrt{n})$ (see Section 1 ) and where $L\left(1 / 2, E^{(n)}\right)$ is the value at the center of the critical strip of the $n$-th twist of a modular elliptic curve $E$ (whose value is predicted by the refined Birch-Swinnerton-Dyer Conjecture). Unfortunately, for $m=4,5$ the integrals of Bump and Ginzburg do not work on the double cover. This is consistent with the complications in higher degree suggested in Section 4 below. 
Similarly, Ginzburg [Gi] has obtained a Rankin-Selberg integral which assembles the Bessel coefficients of an automorphic form on $S O(7)$, which, combining with Theorem 6.1 of Bump, Friedberg, and Furusawa [BFF], should give the series (3.1). However, for $S O(9)$ and beyond no such construction has been found.

As in some of the other cases mentioned in this paper, the details of summing the $G L(3)$ quadratic twists have not yet been carried out. Obstructions include the treatment of the archimedean integrals obtained when one applies the RankinSelberg integrals described above and convergence difficulties to be resolved by truncation. To obtain theorems about the non-vanishing of $L$-values, one must also verify that the residue at a given value of $s$ is non-zero. Overcoming the first of these obstructions may require new information about the (archimedean) Whittaker functions on these groups.

\section{A general conjecture. Applications and related questions}

Based on these examples, it is natural to conjecture that the sum of the quadratic twists of any Langlands $L$-function has meromorphic continuation as a function of $(s, w)$. For expositional purposes, we consider only the standard $L$-functions on $G L(m)$ (according to the functoriality conjecture, this is in fact the general case). As we shall explain below, if $m \geq 4$, the series in two variables is not expected to continue to all of $\mathbb{C}^{2}$, but only to a certain piece.

Once again we work over the base field $\mathbb{Q}$ for simplicity; the general case may be formulated as in $[\mathrm{F}-\mathrm{H}]$. Let $\pi=\otimes \pi_{v}$ be a factorizable automorphic representation of $G L(m)$, and let $L(s, \pi)$ denote the standard $L$-function for $\pi$, described in Section 3 . Suppose for convenience that $\pi$ has unitary central character. Let $S$ be a finite set of finite primes containing 2 and those primes $v$ for which the local representation $\pi_{v}$ is not unramified principal series. If $n=n_{1} n_{2}^{2}$ with $n_{1}, n_{2} \in \mathbb{Z}, n_{1}$ squarefree, then by $L\left(s, \pi, \chi_{n}\right)$ we shall mean the standard twisted $L$-factor $\prod_{v \notin S,\left(v, n_{2}\right)=1} L_{v}\left(s, \pi_{v}, \chi_{n_{1}}\right)$ times a certain finite factor in the primes $v$ dividing $n_{2}$ and those in $S$, depending on $\pi_{v}$ and $\operatorname{ord}_{v}\left(n_{2}\right)$ (the precise dependence of this finite factor on $n_{2}$ may be described by using the formula presented in [BFH4]; it is compatible with the $G L(1)$ situation described in Section 1 and with induction). Define the two functions

$$
Z_{ \pm}(s, w, \pi)=\sum_{ \pm n>0} \frac{L\left(s, \pi, \chi_{n}\right)}{|n|^{w}}
$$

These series converge in the tube domain $\operatorname{Re}(s), \operatorname{Re}(w)>1$.

To describe the continuation of these series, let us introduce a group of transformations $\Gamma_{m}$ of $\mathbb{C}^{2}$ for each $m \geq 1$. By definition, $\Gamma_{m}$ is the group generated by the two involutions

$$
\begin{aligned}
& (s, w) \mapsto(1-s, w+m s-m / 2) \\
& (s, w) \mapsto(s+w-1 / 2,1-w) .
\end{aligned}
$$

It is easy to check that if $m \leq 3$, then $\Gamma_{m}$ is finite. More precisely, if $\mathrm{D}_{n}$ denotes the $n$-th dihedral group $\left(\left|\mathrm{D}_{n}\right|=2 n\right)$, then $\Gamma_{1}=\mathrm{D}_{3}, \Gamma_{2}=\mathrm{D}_{4}$, and $\Gamma_{3}=\mathrm{D}_{6}$. By contrast if $m \geq 4$, then $\Gamma_{m}$ is infinite; indeed it is an affine Weyl group in two generators. Intriguingly, if $W(\mathfrak{g})$ denotes the Weyl group of the Cartan classification type $\mathfrak{g}$, then $\Gamma_{1}=W\left(A_{2}\right), \Gamma_{2}=W\left(B_{2}\right)=W\left(C_{2}\right), \Gamma_{3}=W\left(G_{2}\right)$. For $m=1,2$, the series 
$Z_{ \pm}$may also be obtained by consideration of Eisenstein series on the metaplectic double cover of the corresponding groups $G L_{3}$ and $G S p_{4}$.

Let $R_{m}$ be the region in $(s, w)$-space described as follows. For $m \leq 3, R_{m}=\mathbb{C}^{2}$. For each $m \geq 4$, let $\lambda_{ \pm}(m)$ denote the two numbers

$$
\lambda_{ \pm}(m)=\frac{m \pm \sqrt{m^{2}-4 m}}{2} .
$$

(When $m=4, \lambda_{+}(4)=\lambda_{-}(4)$.) Then, for $m \geq 4$,

$$
R_{m}=\left\{(s, w) \in \mathbb{C}^{2} \mid \operatorname{Re}\left(w+\lambda_{ \pm}(m) s\right)>\frac{\lambda_{ \pm}(m)+1}{2}\right\} .
$$

Note that the region $R_{m}$ is fixed by $\Gamma_{m}$. Then we make the following conjecture.

Conjecture. Each function $Z_{ \pm}$has meromorphic continuation as a function of two complex variables $(s, w)$ to the region $R_{m} \subseteq \mathbb{C}^{2}$. Moreover, each function $Z_{ \pm}$ has a polar divisor stable under $\Gamma_{m}$ and containing the line $w=1$. If $\pi$ is cuspidal, then all poles are simple, and the polar divisor is precisely the image of $w=1$ under $\Gamma_{m}$. The region $R_{m}$ is the largest domain to which the functions $Z_{ \pm}$may be meromorphically extended.

The conjectured behavior of $Z_{ \pm}$is consistent with the known cases described above. One may also formulate a sieved version of the conjecture, where one sums over only those $n$ such that a finite set of finite primes has prescribed behavior in the extension $\mathbb{Q}(\sqrt{n}) / \mathbb{Q}$.

In the remainder of this section we shall motivate this conjecture and explore its consequences. Let us begin with some heuristics pointing to the conjecture.

Let us suppose that $\pi$ is of "level one", that is, that an automorphic form in the space of $\pi$ is invariant under the full modular group $G L(n, \mathbb{Z})$. Then the twisted $L$-function $L\left(s, \pi, \chi_{n}\right)$ satisfies a functional equation

$$
L\left(s, \pi, \chi_{n}\right)=\epsilon\left(s, \pi, \chi_{n}\right) L\left(1-s, \pi, \chi_{n}\right)
$$

where the epsilon-factor in the functional equation satisfies

$$
\epsilon\left(s, \pi, \chi_{n}\right)=|n|^{m(1 / 2-s)} \epsilon(s, \pi) .
$$

(The functional equation (4.2) requires the inclusion of an archimedean factor, but let us ignore this.) Combining (4.2) and (4.3), one arrives at once at the invariance of the series $Z_{ \pm}(s, w)$ under the transformation $(s, w) \mapsto(1-s, w+$ $m s-m / 2)$. Similarly, suppose that the Dirichlet series representing $L\left(s, \pi, \chi_{n}\right)$ is $\sum_{m>0} a_{m} \chi_{n}(m) m^{-s}$. Substituting this expression into $Z_{ \pm}(s, w)$ and interchanging the order of summation, one arrives at the series

$$
\sum_{m>0}\left(\sum_{ \pm n>0} \chi_{n}(m)|n|^{-w}\right) a_{m} m^{-s} .
$$

The inner sum is (essentially) a $G L(1)$ Dirichlet $L$-function in $w$; using its functional equation gives the invariance of the series $Z_{ \pm}$under the transformation $(s, w) \mapsto$ 
$(s+w-1 / 2,1-w)$. Also, the existence of a polar divisor of $w=1$ is suggested by (4.4), since the summand when $m=1$ is just $\zeta(w)$. In fact, this argument is slightly sloppy; after applying the law of quadratic reciprocity, one sees that the precise functional equation requires combining $Z_{+}$and $Z_{-}$; compare (1.3). In general, one should expect an exact functional equation under the transformation $(s, w) \mapsto(s+w-1 / 2,1-w)$ only for a sum of the form $I_{+}\left(s, w, \pi_{\infty}\right) Z_{+}(s, w, \pi)+$ $I_{-}\left(s, w, \pi_{\infty}\right) Z_{-}(s, w, \pi)$ where the $I_{ \pm}\left(s, w, \pi_{\infty}\right)$ are non-zero functions depending in $\pi$ only on $\pi_{\infty}$.

This argument and the examples above suggest the statements concerning the polar divisor. For higher level, though the situation is more complicated, one can make similar arguments, and one should expect the same polar divisor. Moreover, if $m=4$, then there is one line which is pointwise fixed under the maximal infinite subgroup of the group $\Gamma_{4}$ (this subgroup is generated by the product of the two transformations (4.1)); while if $m>4$, then there are exactly two lines which are stable under the maximal infinite subgroup of $\Gamma_{m}$. In all cases, these lines contain the fixed point of $\Gamma_{m},(1 / 2,1 / 2)$. It seems natural to expect continuation to the region bounded by these lines and containing the region of absolute convergence (this region is convex). This is the region $R_{m}$ described above. If $m \leq 3$, then the fixed lines are not given by real equations. However, one may argue, by combining the functional equations in $\Gamma_{m}$ with Hartogs's Theorem concerning continuation to the convex hull of a tube domain (see, for example, [Hor, Theorem 2.5.10]), that the functions $Z_{ \pm}$should have continuation to all of $\mathbb{C}^{2}$; in this sense the heuristics presented here are consistent with the results described in Sections 1-3. It seems likely that these methods can in fact be used to give alternate derivations of the continuations described in those sections, though the technical details have not yet been carried out. Note that the calculation of the residues requires additional information. The combination of the functional equations with Hartogs's Theorem may also be used when $m \geq 4$ to extend the region of meromorphicity of $Z_{ \pm}$beyond the region $\operatorname{Re}(s), \operatorname{Re}(w)>1$, but this approach stops far short of allowing us to establish the conjecture.

Let us add one remark about the conjectured behavior of the polar divisor. One sees easily that the conjecture implies that there are half-lines of poles approaching arbitrarily closely the boundary of the region $R_{m}$. This suggests that the half-lines bounding the region are lines of essential singularities and that they form a natural boundary of the series.

We turn to applications of this conjecture.

The first application is to the non-vanishing of $L$-functions. Suppose that $Z_{ \pm}$has a pole in $w$ for given $s_{0}$. Then one concludes that infinitely many $L\left(s_{0}, \pi, \chi_{n}\right)$ with $\pm n>0$ are non-zero. Similarly, differentiating with respect to $s$, one may prove the non-vanishing of derivatives. Results concerning the non-vanishing of $G L(m) L$ series under finite order twists have been obtained by Barthel and Ramakrishnan [B-R]; however, this method would yield results for quadratic twists and would allow one to establish non-vanishing at the center of the critical strip, which is outside the region given in [B-R]. At the center of the critical strip $s=1 / 2$ the lines of poles occur in intersecting pairs (due to the first functional equation in (4.1) above), and one expects that the condition that the pairs of poles in $w$ not cancel at $s=1 / 2$ will be connected to epsilon-factors (see [BFH2], [F-H]). Moreover, it is an observation of Luo, Rudnick, and Sarnak [LRS] that the non-vanishing of 
twists of the standard $L$-function on $G L(m)$ for all $m$ would imply the RamanujanPetersson conjecture for all automorphic representations of $G L(2)$. (This conjecture states that the Fourier coefficients of $\pi$ are bounded by a particular bound or, equivalently, specifies that no complementary series representations occur as the local constituents $\pi_{v}$. For holomorphic modular forms over $\mathbb{Q}$ it was proved by Deligne, using algebraic geometry.)

There are other variations on this theme. For example, if one could establish the continuation for $\pi$ on $G L(4)$, then one could study the question of the simultaneous non-vanishing of pairs of $L$-series under quadratic twists, as follows. Suppose that $f, g$ are two newforms on $G L(2)$. Then there exists an Eisenstein series on $G L(4)$ whose twisted Whittaker-Fourier coefficients are the products of quadratic twists $L\left(s, f, \chi_{n}\right) L\left(s, g, \chi_{n}\right)$. The conjecture then asserts that one should be able to control the behavior of

$$
\sum_{ \pm n>0} \frac{L\left(s, f, \chi_{n}\right) L\left(s, g, \chi_{n}\right)}{|n|^{w}}
$$

If there exists a quadratic character $\chi_{n}$ with the property that $L\left(s, f, \chi_{n}\right)$ and $L\left(s, g, \chi_{n}\right)$ do not both have negative signs in their functional equations, then one should be able to show that (4.5) has a pole at $w=1$ when $s=1 / 2$. This would imply that there are infinitely many $\chi_{n}$ such that

$$
L\left(1 / 2, f, \chi_{n}\right) L\left(1 / 2, g, \chi_{n}\right) \neq 0 .
$$

To show such a non-vanishing by averaging techniques would require controlling a degree 4 Euler product, something that is well beyond current techniques. As an arithmetic application, in the case that $f$ and $g$ correspond to elliptic curves $E_{f}$, $E_{g}$, the work of Kolyvagin (together with the results described in Section 2) would then imply that there are infinitely many fundamental discriminants $n$ such that the twisted elliptic curves $E_{f}^{(n)}$ and $E_{g}^{(n)}$ both have Mordell-Weil rank zero.

The second application concerns moments and the size of twisted $L$-values. To study these, one once again applies the conjecture in a case when the automorphic representation $\pi$ is not cuspidal. For example, take the case that $\pi$ is the automorphic representation associated to a minimal parabolic Eisenstein series on $G L(m)$. Then, as a special case of a theorem of Jacquet [Ja],

$$
L\left(s, \pi, \chi_{n}\right)=L\left(s, \chi_{n}\right)^{m},
$$

i.e. one obtains the $m$-th power of the original quadratic Dirichlet $L$-series. If $m$ is even, simply obtaining the convergence of $Z_{ \pm}(1 / 2, w, \pi)$ back to $\operatorname{Re}(w)>1$ would imply that, for either choice of sign of $n$,

$$
L\left(1 / 2, \chi_{n}\right) \ll|n|^{\frac{1}{m}+\epsilon} .
$$

In other words, the analytic continuation of this $Z$ for arbitrarily large even $m$ would imply the Lindelöf Hypothesis in $n$-aspect! A similar result may be obtained for odd $m$ under some additional assumptions. Unfortunately $m=3$, accessible by present methods as explained above, is not enough to even break the well-known $1 / 4$ convexity bound for $L\left(1 / 2, \chi_{n}\right)$. 
Note that in both applications mentioned above the crucial matter is the analytic continuation of $Z$ to a neighborhood of $\left(\frac{1}{2}, 1\right)$. This lies inside the region $R_{m}$, and it is for this reason tha the conjecture would have such interesting consequences.

We remark that since $\pi$ here is not cuspidal, one expects that the poles in $(s, w)$ should coalesce when $s=1 / 2$ to give a higher order pole in $w$. In fact one expects that each of the series $\sum_{ \pm n>0} L\left(1 / 2, \chi_{n}\right)^{m}|n|^{-w}$, absolutely convergent for $\operatorname{Re}(w)>1+\frac{3}{16} m$ by the bound of Burgess [Bu], has a pole of order $1+\left(m^{2}+m\right) / 2$ at $w=1$. Perhaps more surprising is that the heuristics described above predict that these series behave very differently to the left of $w=1$ for different values of $m$. That is,

Conjecture. For each choice of sign, the series

$$
\sum_{ \pm n>0} L\left(1 / 2, \chi_{n}\right)^{m}|n|^{-w}
$$

has meromorphic continuation to the entire complex plane when $m \leq 3$, while it has continuation up to an essential singularity at $w=1 / 2$ for $m \geq 4$.

Similarly, the general conjecture would give control over quantities such as

$$
\sum_{ \pm n>0,|n|<x} L\left(s_{0}, \pi, \chi_{n}\right)^{m} .
$$

For $\pi$ on $G L(2)$, this quantity is related to the constants arising in Szpiro's conjecture (see Goldfeld-Szpiro [G-S]).

One may also formulate a conjecture for a series in more than two complex variables, such as the sum

$$
\sum \frac{L\left(s_{1}, \pi_{1}, \chi_{n}\right) \cdots L\left(s_{m}, \pi_{m}, \chi_{n}\right)}{|n|^{w}}
$$

Similar heuristics show that the group of functional equations is expected to be finite only if the total degree of the Euler product in the numerator is at most 3 . A few additional finite groups arise in these low-degree cases.

To conclude this section, let us pose an additional question. Automorphic $L$ functions may conjecturally be used to construct the zeta-functions associated to algebraic varieties defined over number fields or function fields. Are there algebreogeometric analogues of this conjecture? Is there a natural construction for $\ell$-adic representations? In the case that $\pi$ is the automorphic representation corresponding to a holomorphic cusp form $f$ of weight 2 , so that the $L$-function for $f$ is that of an elliptic curve $E$ defined over $\mathbb{Q}$, do the series $Z_{ \pm}$have a natural meaning in terms of $E$ ? In the function field case, is there a connection to the arithmetic of arithmetic surfaces?

\section{Higher twists}

In this last section, we present yet another variation on the basic theme described here. Namely, in some instances the quadratic characters $\chi_{n}$ of Sections 1-4 may be replaced by characters of higher order! That is, one may consider the sum of 
cubic or higher-order twists of a given $L$-function. The constructions to date which do this make use of the higher metaplectic covers of groups such as $G L(n)$. These covers are constructed using a 2-cocycle whose properties are closely tied to the higher reciprocity laws studied by Legendre, Gauss, Hilbert, Hasse and others. For a survey, see [Ho, Section 2]; for a detailed treatment, see Kazhdan-Patterson [K-P] or Matsumoto [Mat].

Let us begin with $G L(1)$. If $\chi$ is on $G L(1)$ and the base field contains the $r$-th roots of unity, then for any $r$ it is possible to obtain the twists of $\chi$ by all its $r$-th order characters as the Whittaker-Fourier coefficients of an Eisenstein series on the $r$-cover of $G L(r)$. When $r=2$, this is the content of our first example above. When $r=3$, this was demonstrated by Bump and Hoffstein in [B-H]. For $r \geq 4$, it was demonstrated in an ad hoc way by Bump and Hoffstein (see [Ho, Section 5]; the proofs there are incomplete), while work in progress of Bump and Lieman seeks to develop a satisfactory theory based on the uniqueness of Whittaker models which explains why the phenomenon occurs [B-L].

By taking an integral transform of this Eisenstein series on the $r$-cover of $G L(r)$, one can obtain the analytic continuation and functional equation of a Dirichlet series whose coefficients are $r$-th order $L$-series. For example, if the character $\chi$ on $G L(1)$ is the trivial one, then one obtains the analytic continuation and functional equation (in $w$ ) of

$$
\sum \frac{L_{r}\left(s, \chi_{n}^{(r)}\right)}{\mathrm{N} n^{w}}
$$

where $n$ varies over the non-zero integers in the ground field, $\mathrm{N}$ denotes the absolute norm, and

$$
\chi_{n}^{(r)}(m)=\left(\frac{n}{m}\right)_{r}
$$

is given by the value of an $r$-th order power residue symbol. The subscript $r$ in the expression $L_{r}$ indicates that the Euler factors at the primes dividing $r$ must be removed. By applying analytic techniques, one may then obtain mean value results for $\sum_{\mathrm{N} n<x} L_{r}\left(s, \chi_{n}^{(r)}\right)$. In the case $r=3$ this is being done by Farmer, Hoffstein, and Lieman (the work was begun long ago by Hoffstein but became bogged down in many difficult analytic details). The functional equation in $w$ in this last case interchanges $L_{3}\left(s, \chi_{n}^{(3)}\right)$ and $L_{3}\left(s, \chi_{n^{2}}^{(3)}\right)$. Note that an attempt to use a Siegel type direct averaging technique will not succeed for $s$ close to $1 / 2$, as the approximate functional equation gets affected by the argument of the cubic Gauss sums.

By taking non-trivial $\chi$, one can sum higher twists of Hecke $L$-series (compare (1.4)). In certain instances these correspond to a family of elliptic curves. For example, the $L$-series of the family of CM elliptic curves $x^{3}+y^{3}=D$ are the cubic twists of a Hecke $L$-series with Grössencharacter defined over the field $\mathbb{Q}(\sqrt{-3})$. In [Li2], Lieman uses this approach to deduce non-vanishing theorems for subclasses of these $L$-series. By the theorem of Coates-Wiles, this non-vanishing implies that for infinitely many such $D$ the corresponding elliptic curves have only finitely many rational points.

Another example involving higher twists, this time of a $G L(2) L$-series, is obtained by generalizing the construction (2.3). One may convolve an Eisenstein series on the $r$-fold cover of $G L(2)$ with $f$, a non-metaplectic form on $G L(2)$, provided that the ground field contains the $r$-th roots of unity. This produces the analytic 
continuation of something of the form

$$
D(s, w)=\sum_{n} \frac{S(w, n) \overline{c_{n}}}{\mathrm{~N} n^{s}}
$$

where

$$
S(w, n)=\sum_{d} \frac{G(n, d)}{\mathrm{N} d^{w}}
$$

is a Dirichlet series built out of the $r$-th order Gauss sums

$$
G(n, d)=\frac{1}{\sqrt{\mathrm{Nd}}} \sum_{\alpha \bmod d}\left(\frac{\alpha}{d}\right)_{r} e\left(\operatorname{tr}\left(\frac{n \alpha}{d}\right)\right),
$$

with tr denoting the absolute trace and $c_{n}$ denoting the Fourier coefficients of $f$. Interchanging the order of summation, one gets

$$
D(s, w)=\sum_{d} \frac{G(1, d) L\left(s, \bar{f}, \tilde{\chi}_{d}^{(r)}\right)}{\mathrm{N} d^{w}}
$$

where the $L$-series in the numerator is the twist of the $L$-series of $\bar{f}$ by the $r$-th order character $\tilde{\chi}_{d}^{(r)}(m)=\left(\frac{m}{d}\right)_{r}$. For fixed $s$, it may be shown that $D(s, w)$ has poles at $w=\frac{1}{2}+\frac{1}{r}$ and at $w=\frac{3}{2}-2 s+\frac{1}{r}$. Thus, for example, at $s=1 / 2$ these poles coalesce at $w=1 / 2+1 / r$. The residue at $w=1 / 2+1 / r$ is an interesting new object: $L\left(s+1 / 2 r, \bar{f} \times \theta_{r}\right)$, that is, the Rankin-Selberg convolution of $\bar{f}$ with $\theta_{r}$, the theta function on the $r$-fold cover of $G L(2)$, evaluated at the point $s+1 / 2 r$. If it could be proved that at this special value the Rankin-Selberg convolution does not vanish, then the pole would exist, and it would follow that $L\left(s, \bar{f}, \tilde{\chi}_{d}^{(r)}\right) \neq 0$ infinitely often. Unfortunately, this is a challenging problem, for the Rankin-Selberg convolutions in question do not have Euler products and moreover, except for the cases $r=2,3$, the $\theta_{r}$ are not well understood. In particular instances this non-vanishing has been established for $r=3$ by She [Sh], using a combination of computer calculation and theoretical arguments.

Though the study of higher-order twists is not well developed, there is one other construction involving the twists of $G L(2)$ automorphic $L$-series. A way has been found to convolve the theta function on the 3-fold cover of $G L(3)$ with a cusp form $f$ on $G L(2)$. This construction, discovered by Bump, Ginzburg and Hoffstein [BGH], was used to express the symmetric cube $L$-series of $f$ as a Rankin-Selberg integral. The same Rankin-Selberg construction should work if one replaces the cubic theta function by the maximal parabolic Eisenstein series on the triple cover of $G L(3)$ considered by Bump and Hoffstein [B-H]. If one did this and applied the same "interchange in the order of summation" argument, then one would probably obtain the analytic continuation of

$$
\sum \frac{L\left(s, \bar{f}, \tilde{\chi}_{d}^{(3)}\right)}{\mathrm{N} d^{w}}
$$

where the $L$-series are cubic twists of the $L$-series for $\bar{f}$, i.e. the same series as (5.1) but with the Gauss sums removed. If $s=1 / 2$, the residues of the poles in $w$ would 
then be proportional to the symmetric cube $L$-series at the point $3 / 2$, and these would certainly not vanish. This construction also provides some evidence that the double Dirichlet series in $(s, w)$ has a finite group of functional equations.

It appears that this is the only remaining example where a finite group of more than two functional equations is to be expected. In other cases of higher twists, the heuristics described in Section 4 do not carry over, since the functional equations of the series $L\left(s, \chi_{n}^{(r)}\right)$ involve higher-order Gauss sums, and this prevents an interchange of summation from giving a functional equation in $(s, w)$. One is left with simply posing the following question:

Fix $r>2$, and let $\pi=\otimes \pi_{v}$ be a factorizable automorphic representation of a connected reductive linear algebraic group $G$ over a global field $F$ containing the $r$-th roots of unity. Let $\rho$ be a finite-dimensional representation of the $L$-group of $G$. If $\chi$ is an idele class character of $F$, let $L(s, \pi, \rho, \chi)$ denote the twist of the Langlands $L$-function $L(s, \pi, \rho)$ by $\chi$; this is a product of local factors $L_{v}$. We suppose for convenience that the ring of integers $\mathcal{O}$ of $F$ has class number one; the general case may be formulated as in $[\mathrm{F}-\mathrm{H}]$. Let $\mathrm{N}$ denote the absolute norm. Let $S$ be a finite set of finite primes containing those dividing $r$ and those for which the local representation $\pi_{v}$ is not unramified principal series. If $n=n_{1} n_{2}^{r}$ with $n_{1}, n_{2} \in \mathcal{O}, n_{1} r$-th power free, then by $L\left(s, \pi, \rho, \chi_{n}^{(r)}\right)$ we shall mean the twisted $L$-factor $\prod_{v \notin S,\left(v, n_{2}\right)=1} L_{v}\left(s, \pi_{v}, \rho, \chi_{n_{1}}^{(r)}\right)$ times a certain finite factor in the primes $v$ dividing $n_{2}$ and those in $S$, depending on $\pi_{v}$ and $\operatorname{ord}_{v}\left(n_{2}\right)$ (the precise dependence on the primes $v \mid n_{2}$ is specified by requiring that these factors be consistent with the $G L(1)$ case above when the representation is unramified principal series).

Question. Define the function

$$
Z^{(r)}(s, w, \pi, \rho)=\sum_{n \in \mathcal{O} /\left(\mathcal{O}^{\times}\right)^{r}} L\left(s, \pi, \rho, \chi_{n}^{(r)}\right) N n^{-w}
$$

convergent for $\operatorname{Re}(s), \operatorname{Re}(w)$ sufficiently large. To what region of $(s, w)$ space does this function have meromorphic continuation? What is its precise polar divisor?

The construction described here may be regarded as roughly summing over all cyclic extensions of $F$ of order $r$; one sums the twist of $L(s, \pi)$ by the automorphic object associated by class field theory to each such extension, divided by a complex power of its conductor. One may also ask if there are analogous constructions for other extensions of $F$ of fixed Galois group which admit non-trivial continuation.

\section{REFERENCES}

[Ar] J. Arthur, A trace formula for reductive groups II: Applications of a truncation operator, Compos. Math. 40 (1980), 87-121. MR 81b:22018

[B-R] L. Barthel and D. Ramakrishnan, A non-vanishing result for twists of L-functions of GL(n), Duke Math. J. 74 (1994), 681-700. MR 95d:11062

[Bo] A. Borel, Automorphic L-functions, in Automorphic Forms, Representations and Lfunctions, Proc. Symp. Pure Math. 33, part 2, A.M.S., Providence, 1979, pp. 27-61. MR 81m:10056

[Bump] D. Bump, The Rankin-Selberg method: A survey, in Number Theory, Trace Formulas, and Discrete Groups (K. Aubert, E. Bombieri, D. Goldfeld, eds.), Academic Press, Inc., Boston, 1989, pp. 49-109. MR 90m:11079

$[\mathrm{BFF}]$ D. Bump, S. Friedberg, and M. Furusawa, Explicit formulas for the Waldspurger and Bessel models, preprint. 
[BFH1] D. Bump, S. Friedberg, and J. Hoffstein, A nonvanishing theorem for derivatives of automorphic L-functions with applications to elliptic curves, Bull. A. M. S. (N.S.) 21 (1989), 89-93. MR 90b:11063

[BFH2] _ Eisenstein series on the metaplectic group and nonvanishing theorems for automorphic L-functions and their derivatives, Annals of Math. 131 (1990), 53-127. MR 92e: 11053

[BFH3] , Nonvanishing theorems for L-functions of modular forms and their derivatives, Inventiones Math. 102 (1990), 543-618. MR 92a:11058

[BFH4] , p-adic Whittaker functions on the metaplectic group, Duke Math. J. 63 (1991), 379-397. MR 92d:22024

[B-G] D. Bump and D. Ginzburg, Spin L-functions on the symplectic group, International Math. Res. Notices 8 (1992), 153-160. MR 93i:11060

[BGH] D. Bump, D. Ginzburg, and J. Hoffstein, The symmetric cube, To appear in Inventiones Math.

[B-H] D. Bump and J. Hoffstein, Cubic metaplectic forms on GL(3), Inventiones Math. 84 (1986), 481-505. MR 87i:11059

[B-L] D. Bump and D. Lieman, Uniqueness of Whittaker functionals on the metaplectic group, Duke Math. J. 76 (1994), 731-739. MR 309:328

[Bu] D. Burgess, On character sums and L-series, II, Proc. London Math. Soc. 13 (1963), 524-536. MR 26:6133

[F-H] S. Friedberg and J. Hoffstein, Nonvanishing theorems for automorphic L-functions on $G L(2)$, Annals of Math. 142 (1995), 385-423. MR 343:325

[Fu] M. Furusawa, On the theta lift from $S O_{2 n+1}$ to $\widetilde{S p}_{n}$, J. Reine Angew. Math. 466 (1995), 87-110.

[Ge] S. Gelbart, An elementary introduction to the Langlands program, Bull. A. M. S. (N.S.) 10 (1984), 177-219. MR 85e:11094

[G-P] S. Gelbart and I. Piatetski-Shapiro, L-Functions for $G \times G L(n)$, in Explicit Constructions of $L$-functions, Springer Lecture Notes \#1254, Springer-Verlag, 1987. MR 89k:11038

[Gi] D. Ginzburg, Fax to Daniel Bump (1994).

[G-H] D. Goldfeld and J. Hoffstein, Eisenstein series of $1 / 2$-integral weight and the mean value of real Dirichlet L-series, Inventiones Math. 80 (1985), 185-208. MR 86m:11029

[G-S] D. Goldfeld and L. Szpiro, Bounds for the order of the Tate-Shafarevich group, Compos. Math. 97 (1995), 71-87. MR 1:355 118

[Gu] S. Gupta, Average value of quadratic twists of L-functions over function fields, Thesis, Brown University (1995).

[HST] M. Harris, D. Soudry, and R. Taylor, $l$-adic representations associated to modular forms over imaginary quadratic fields I: Lifting to $G S p_{4}(\mathbb{Q})$, Inventiones Math. 112 (1993), 377-411. MR 94d:11035

[Ho] J. Hoffstein, Eisenstein series and theta functions on the metaplectic group, CRM Proceedings and Lecture Notes, vol. 1, A.M.S., Providence, 1993, pp. 65-104. MR 94h:11050

[Hor] L. Hormander, An introduction to complex analysis in several variables, 3rd rev. ed., North-Holland, Amsterdam, 1990. MR 91a:32001

[Iw] H. Iwaniec, On the order of vanishing of modular L-functions at the critical point, Séminaire de Théorie des Nombres, Bordeaux 2 (1990), 365-376. MR 92h:11040

[Ja] H. Jacquet, Principal L-functions of the linear group, in Automorphic Forms, Representations and $L$-Functions, Proc. Symp. Pure Math. 33, part 2, A.M.S., Providence, 1979, pp. 63-86. MR 81f:22029

[Ju] M. Jutila, On the mean value of $L(1 / 2, \chi)$ for real characters, Analysis 1 (1981), 149-161. MR 82m: 10065

[K-P] D. Kazhdan and S. Patterson, Metaplectic forms, Publ. Math. I.H.E.S. 59 (1984), 35-142. MR 85g:22033

[Ko] V. Kolyvagin, On the Mordell-Weil group and the Shafarevich-Tate group of Weil elliptic curves, Izv. Akad. Nauk SSSR Ser. Mat. 52 (1988), 1154-1180. MR 90f:11035

[La] R. Langlands, Problems in the theory of automorphic forms, in Lectures in Modern Analysis and Applications, Springer Lecture Notes \#170, Springer-Verlag, 1970, pp. 1886. MR 46: 1758 
[Li1] D. Lieman, The GL(2) Rankin-Selberg convolution for higher level non-cuspidal forms, Contemp. Math. 166 (1994), 83-92. MR 95h:11041

[Li2] Nonvanishing of L-series associated to cubic twists of elliptic curves, Annals of Math. 140 (1994), 81-108. MR 95g:11044

[LRS] W. Luo, Z. Rudnick, and P. Sarnak, On Selberg's eigenvalue conjecture, Geom. Funct. Anal. 5 (1995), 387-401. MR 1:334 872

[Ma] H. Maass, Konstruktion ganzer Modulformen halbzahliger Dimension, Abh. Math. Semin. Univ. Hamburg 12 (1937), 133-162.

[Mat] H. Matsumoto, Sur les sous-groupes arithmétiques des groupes semi-simples déployés, Ann. Scient. Ec. Norm. Sup. (1969), 1-62. MR 39:1566

[M-M] K. Murty and R. Murty, Mean values of derivatives of modular L-series, Annals of Math. 133 (1991), 447-475. MR 92e:11050

[No] M. Novodvorsky, Automorphic L-functions for the group GSp $p_{4}$, in Automorphic Forms, Representations and $L$-functions, Proc. Symp. Pure Math. 33, part 2, A.M.S., Providence, 1979, pp. 87-95. MR 81c:10032

[Sh] X. She, On the nonvanishing of cubic twists of automorphic L-series, Thesis, Brown University (1995).

[Shi] G. Shimura, On modular forms of half-integral weight, Annals of Math. 97 (1973), 440481. MR 48:10989

[Si] C. L. Siegel, The average measure of quadratic forms with given determinant and signature, in Gesammelte Abhandlungen, Band II, Springer-Verlag, 1966, pp. 473-491.

[Ta] R. Taylor, l-adic representations associated to modular forms over imaginary quadratic fields II, Inventiones Math. 116 (1994), 619-643. MR 95h:11050a

[Za] D. Zagier, The Rankin-Selberg method for automorphic forms which are not of rapid decay, J. Fac. Sci. Univ. Tokyo, Sect. IA (Math.) 28 (1981), 415-437. MR 83k:10056

Department of Mathematics, Stanford University, Stanford, CA 94305-2125

E-mail address: bump@gauss.stanford.edu

Department of Mathematics, University of California Santa Cruz, Santa Cruz, CA 95064

E-mail address: friedbe@cats.ucsc.edu

Department of Mathematics, Brown University, Providence, RI 02912

E-mail address: jhoff@gauss.math.brown.edu 